\title{
Effective Solution of Riemann Problem for Fifth Order Improperly Elliptic Equation on a Rectangle
}

\author{
Seyed Mohammadali Ali Raeisian \\ Institute of Mathematics, Armenian National Academy of Sciences, Yerevan, Armenia \\ Email: s_ma_raissian@yahoo.com
}

Received August 12, 2012; revised October 17, 2012; accepted October 31, 2012

\begin{abstract}
In this paper we present a numerical method for solving Riemann type problem for the fifth order improperly elliptic equation in complex plane. We reduce this problem to the boundary value problems for properly elliptic equations, and then solve those problems by the grid methods.
\end{abstract}

Keywords: Polyharmonic Equation; Boundary Value Problem; Dirichlet Problem; Improperly Elliptic Equation; Riemann Problem; Grid Method

\section{Introduction}

Let $D$ be rectangle $D=\{(x, y) \mid a<x<b, c<y<d\}$ in a complex plane with boundary $\Gamma=\partial D$.

In this paper, we consider the elliptic equation:

$$
\begin{aligned}
& \frac{\partial^{5}}{\partial \bar{z}^{3} \partial z^{2}} u(x, y) \equiv \frac{1}{2^{5}}\left(\frac{\partial}{\partial x}+\mathrm{i} \frac{\partial}{\partial y}\right)^{3}\left(\frac{\partial}{\partial x}-\mathrm{i} \frac{\partial}{\partial y}\right)^{2}=0, \\
& (x, y) \in D
\end{aligned}
$$

The number of roots of the characteristic equation corresponding to Equation (1.1) in the upper half-plane and lower half plane are not equal therefore it is an improperly elliptic equation and general form of this kind of equations was investigated in [1] in the form of: ${ }^{* * *}$

$$
\begin{aligned}
& \frac{\partial^{n}}{\partial \bar{z}^{p} \partial z^{n-p}} u(x, y) \\
& \equiv \frac{1}{2^{n}}\left(\frac{\partial}{\partial x}+\mathrm{i} \frac{\partial}{\partial y}\right)^{p}\left(\frac{\partial}{\partial x}-\mathrm{i} \frac{\partial}{\partial y}\right)^{n-p} u(x, y)=0,(x, y) \in D_{1}
\end{aligned}
$$

where $D_{1}=\left\{(x, y) \mid x^{2}+y^{2}<1\right\}$ is a unit disk in a complex plane with boundary $\partial D_{1}$. As all classical boundary conditions for improperly elliptic equations are not correct [1]; so we take following boundary conditions for problem (1.1):

$$
\left.u\right|_{\Gamma}=f_{0},\left.\frac{\partial u}{\partial N}\right|_{\Gamma}=f_{1}, \mathfrak{R}\left(\frac{\partial^{2} u}{\partial N^{2}}\right)=f_{2},
$$

where $f_{0} \in C^{(2, \alpha)}(\Gamma), f_{1} \in C^{(1, \alpha)}(\Gamma), f_{2} \in C^{(\alpha)}(\Gamma)$,

(i.e. a function $f_{0}$ with second order derivative $\frac{\mathrm{d}^{2} f_{0}}{\mathrm{~d} s^{2}}$ satisfy Holder condition on $\Gamma, f_{1}$ with first order derivative satisfy Holder condition on $\Gamma$ and $f_{2}$ satisfy Holder condition on $\Gamma$ ) are given functions on $\Gamma$. We are seeking the solution of (1.1), (1.2) in the class of functions $C^{(5)}(D) \cap C^{(2, \alpha)}(D \cup \Gamma)$. The problem (1.1), (1.2) known as "Riemann Dirichlet type problem for fifth order improperly elliptic equation". The general form of this kind of problem was considered in [1] and the solvability of non-homogeneous problem and general solution of corresponding homogeneous problem was discussed there. The number of corresponding linearly independent solution of homogeneous problem in our case is one (see [1]) therefore the problem is not uniquely solvable and on the other hand we are dealing with complex domain which makes more complicated obstacles in realizing ideas. Although there was a few limited efforts in the especial cases like first order improperly and second order properly elliptic equations to present as effecttive finite difference method in [2] but generally speaking; there is not too much practical suitable methods for higher order improperly elliptic equations. So, in this paper, our strategy based on converting boundary value problem for elliptic equation with complex coefficients into real coefficients cases; which could be solve by many fast finite difference methods $([3,4])$.

\section{Description of the Algorithm}

The general solution of (1.1) can be represented in the form:

$$
u=\bar{z}^{2} \phi_{0}(z)+\bar{z} \tilde{\phi}_{1}(z)+z \tilde{\phi}_{2}(\bar{z})+\tilde{\phi}_{3}(z)+\tilde{\phi}_{4}(\bar{z}),
$$

where $\phi_{0}, \tilde{\phi}_{i} \quad(1 \leq i \leq 4)$ are arbitrary analytic functions 
in $D$

So, by using Taylor's expansion, we may replace:

$$
\tilde{\phi}_{1}(z)=C_{1}+z \phi_{1}(z), \tilde{\phi}_{2}(\bar{z})=C_{2}+\bar{z} \phi_{2}(\bar{z}),
$$

Then, we will get:

$$
\begin{aligned}
u \equiv \bar{z}^{2} \phi_{0}(z)+C_{1} \bar{z}+z \bar{z} \phi_{1}(z)+C_{2} z \\
+z \bar{z} \phi_{2}(\bar{z})+\tilde{\phi}_{3}(z)+\tilde{\phi}_{4}(\bar{z}),
\end{aligned}
$$

or

$$
\begin{aligned}
u & =\bar{z}^{2} \phi_{0}(z)+z \bar{z}\left(\phi_{1}(z)+\phi_{2}(\bar{z})\right) \\
& +\left(\Phi_{3}(z)+\Phi_{4}(\bar{z})\right),
\end{aligned}
$$

On the other hand from (1.1) we get:

$$
\begin{aligned}
& \frac{\partial^{5} \bar{u}}{\partial z^{3} \partial \bar{z}^{2}}=0, \\
& \Rightarrow \frac{\partial^{6} U}{\partial \bar{z}^{3} \partial z^{3}}=\frac{\partial^{6}\left(\frac{u+\bar{u}}{2}\right)}{\partial \bar{z}^{3} \partial z^{3}}=\frac{1}{2}\left(\frac{\partial^{6} u}{\partial z^{3} \partial \bar{z}^{3}}+\frac{\partial^{6} \bar{u}}{\partial z^{3} \partial \bar{z}^{3}}\right)=0,
\end{aligned}
$$

If we denote $U=\Re u$, we have a Dirichlet problem for the determination of $U$ :

$$
\begin{aligned}
& \Delta^{3} U=0,\left.U\right|_{\Gamma}=\left.\mathfrak{R}\left(f_{0}\right)\right|_{\Gamma},\left.\frac{\partial U}{\partial N}\right|_{\Gamma}=\left.\mathfrak{R}\left(f_{1}\right)\right|_{\Gamma}, \\
& \left.\frac{\partial^{2} U}{\partial N^{2}}\right|_{\Gamma}=\left.\mathfrak{R}\left(f_{2}\right)\right|_{\Gamma},
\end{aligned}
$$

The solvability and smoothness of the solution of problem (2.2) follows from the general theory of elliptic problems [5]. From the unique solution of Dirichlet problem for third harmonic Equation (2.2) we have $U$ on all mesh points and a formula for representing unique solution of above Dirichlet problem may be found in [6, p. 149].

Applying bi-harmonic operator $\Delta^{2}$ on real part of $u$ in (2.1); we will get: ${ }^{* *}$

$$
\begin{aligned}
2 \Delta^{2} U & =2 \Delta\left[\Delta\left(\bar{z}^{2} \phi_{0}(z)+z^{2} \overline{\phi_{0}(z)}\right)\right] \\
& =\Delta\left(2 \bar{z} \phi_{0}^{\prime}(z)+2 z \overline{\phi_{0}^{\prime}(z)}+(z \Phi(z))^{\prime}+\overline{(z \Phi(z))^{\prime}}\right) \\
& =2 \phi_{0}^{\prime \prime}(z)+2 \overline{\phi_{0}^{\prime \prime}(z)}=4 \Re\left(\phi_{0}^{\prime \prime}(z)\right),
\end{aligned}
$$

Therefore

$$
\Delta^{2} U=2 \Re\left(\phi_{0}^{\prime \prime}(z)\right),
$$

where $\phi_{0}^{\prime \prime}$ is analytic in $D$, so $\mathfrak{R} \phi_{0}^{\prime \prime}(z)$ is harmonic function.

From (2.3) we have:

$$
\frac{1}{2} \Delta^{2} U=\mathfrak{R}\left(\phi_{0}^{\prime \prime}(z)\right)=\frac{\partial}{\partial x}\left(\mathfrak{R} \phi_{0}^{\prime}(z)\right)=\frac{\partial}{\partial y}\left(\Im \phi_{0}^{\prime}(z)\right),
$$

Denoting $\quad u_{1}=\mathfrak{R}\left(\phi_{0}^{\prime}(z)\right)$ and $v_{1}=\mathfrak{I}\left(\phi_{0}^{\prime}(z)\right)$, we have Poincare problems for the determination of these functions:

$$
\Delta u_{1}=0,\left.\left(\frac{\partial u_{1}}{\partial x}\right)\right|_{\Gamma}=\frac{1}{2}\left(\Delta^{2} U\right)
$$

and

$$
\Delta v_{1}=0,\left.\left(\frac{\partial v_{1}}{\partial y}\right)\right|_{\Gamma}=\frac{1}{2}\left(\Delta^{2} U\right)
$$

Solving these problems, we get: $u_{1}=u_{1}^{0}+c_{1} y+c_{2}$ and $v_{1}=v_{1}^{0}+c_{3} x+c_{4}$ where $u_{1}^{0}, v_{1}^{0}$ are uniquely determined functions and $c_{j}(j=1, \cdots, 4)$ are arbitrary real constants.

We must mention that by Cauchy-Riemann equations we have: $c_{1}=-c_{3}$,therefore we get a representation:

$$
\phi_{0}^{\prime}=\Phi^{0}+C_{1}-\mathrm{i} c_{0} z
$$

Here $C_{1}$ is complex and $c_{0}$ is real arbitrary constants and $\Phi^{0}=u_{1}^{0}+\mathrm{i} v_{1}^{0}$ is uniquely determined analytic function.

By integration we have:

$$
\phi_{0}(z)=w_{0}(z)+\mathrm{i} C_{0} z^{2}+C_{1} z+C_{2},
$$

where $C_{0}$ is real constant, $C_{1}, C_{2}$ are arbitrary complex constants, and $w_{0}$ is uniquely determined function.

Now, replacing $\phi_{0}$ in (2.1), we get:

$$
\begin{aligned}
u= & \bar{z}^{2} w_{0}+\mathrm{i} C_{0}(z \bar{z})^{2}+\left(z \bar{z} \mathfrak{R} \Omega_{1}(z)+\mathfrak{R} \Omega_{2}(z)\right) \\
& +\mathrm{i}\left(z \bar{z} \mathfrak{\Im} \Omega_{3}(z)+\mathfrak{\Im} \Omega_{4}(z)\right)
\end{aligned}
$$

where

$$
\begin{aligned}
& \Omega_{1}(z)=\bar{C}_{1} z+\phi_{1}(z)+\overline{\phi_{2}(\bar{z})} \\
& \Omega_{2}(z)=\bar{C}_{2} z^{2}+\Phi_{3}(z)+\Phi_{4}(z) \\
& \Omega_{3}(z)=-\bar{C}_{1} z+\phi_{1}(z)-\overline{\phi_{2}(\bar{z})} \\
& \Omega_{4}(z)=\left(-\bar{C}_{2} z^{2}+\Phi_{3}(z)-\Phi_{4}(z)\right)
\end{aligned}
$$

are arbitrary analytic functions.

Finally, we represent the solution in the form:

$$
u=\bar{z}^{2} w_{0}(z)+\mathrm{i} C_{0}(z \bar{z})^{2}+H(z, \bar{z})+\mathrm{i} h(z, \bar{z}),
$$

where $H(z, \bar{z})=z \bar{z} \Re \Omega_{1}(z)+\Re \Omega_{2}(z)$ and $h(z, \bar{z})=z \bar{z} \Im \Omega_{3}(z)+\Im \Omega_{4}(z)$. Here $w_{0}$ is known function, and $H, h$ are real valued functions which satisfy the condition $\Delta^{2} H=\Delta^{2} h=0$ and constant $C_{0}$ is arbitrary real constant.

Now, we must determine real valued functions $H, h$. These functions satisfy bi-harmonic equation:

$\Delta^{2} H=\Delta^{2} h=0$ and from (2.9): 


$$
U=\mathfrak{R}\left(\bar{z}^{2} w_{0}(z)\right)+H(x, y),
$$

Hence, we have following Dirichlet conditions on the boundary $\Gamma$ :

$$
\begin{aligned}
& \left.H\right|_{\Gamma}=\mathfrak{R}\left(f_{0}\right)-\left.\mathfrak{R}\left(\bar{z}^{2} w_{0}(z)\right)\right|_{\Gamma}, \\
& \left.\frac{\partial H}{\partial N}\right|_{\Gamma}=\mathfrak{R}\left(f_{1}\right)-\left.\frac{\partial}{\partial N} \mathfrak{R}\left(\bar{z}^{2} w_{0}(z)\right)\right|_{\Gamma},
\end{aligned}
$$

Finally we get the "Dirichlet problem for bi-harmonic equation":

$$
\begin{aligned}
& \Delta^{2} H=0, \\
&\left.H\right|_{\Gamma}= \Re\left(f_{0}\right)-\left.\mathfrak{R}\left(\bar{z}^{2} w_{0}(z)\right)\right|_{\Gamma}, \\
&\left.\frac{\partial H}{\partial N}\right|_{\Gamma}=\mathfrak{R}\left(f_{1}\right)-\left.\frac{\partial}{\partial N} \mathfrak{R}\left(\bar{z}^{2} w_{0}(z)\right)\right|_{\Gamma},
\end{aligned}
$$

which has a unique solution.

Analogously, we get the same boundary problem:

$$
\mathfrak{J} u=\mathfrak{I}\left(\bar{z}^{2} w_{0}(z)\right)+C_{0}(z \bar{z})^{2}+h(x, y),
$$

i.e.:

$$
h(x, y)=\mathfrak{J} u-\mathfrak{J}\left(\bar{z}^{2} w_{0}(z)\right)+C_{0}(z \bar{z})^{2},
$$

for determination of function $h$. This problem includes arbitrary constant $C_{0}$, therefore must be modified.

So, first we find the function $h_{0}$, by solving "Dirichlet problem for bi-harmonic equation":

$$
\begin{aligned}
& \Delta^{2} h_{0}=0, \\
& \left.h_{0}\right|_{\Gamma}=\left.(z \bar{z})^{2}\right|_{\Gamma}, \\
& \left.\left(\frac{\partial}{\partial N} h_{0}\right)\right|_{\Gamma}=\left.\left(\frac{\partial}{\partial N}(z \bar{z})^{2}\right)\right|_{\Gamma},
\end{aligned}
$$

and then ,we solve:

$$
\begin{aligned}
& \Delta^{2} h_{1}=0, \\
& \left.h_{1}\right|_{\Gamma}=\left.(\mathfrak{I} u)\right|_{\Gamma}-\left.\mathfrak{I}\left(\bar{z}^{2} w_{0}(z)\right)\right|_{\Gamma} \\
& =\mathfrak{I} f_{0}-\left.\mathfrak{I}\left(\bar{z}^{2} w_{0}(z)\right)\right|_{\Gamma}, \\
& \left.\left(\frac{\partial h_{1}}{\partial N}\right)\right|_{\Gamma}=\left.\left(\frac{\partial}{\partial N} \mathfrak{I} u\right)\right|_{\Gamma}-\left.\frac{\partial}{\partial N} \mathfrak{I}\left(\bar{z}^{2} w_{0}(z)\right)\right|_{\Gamma} \\
& =\mathfrak{I} f_{1}-\left.\left(\frac{\partial}{\partial N} \mathfrak{I}\left(\bar{z}^{2} w_{0}(z)\right)\right)\right|_{\Gamma},
\end{aligned}
$$

These problems are uniquely solvable, and the solution of (2.11) will be $h=h_{1}-C_{0} h_{0}$.

Replacing the function $h$ into (2.9), we find:

$$
\begin{aligned}
& u=\bar{z}^{2} w_{0}(z)+H(x, y) \\
& +\mathrm{i}\left(h_{1}(x, y)-C_{0} h_{0}(x, y)\right)+\mathrm{i} C_{0}(z \bar{z})^{2},
\end{aligned}
$$

or:

$$
u=\bar{z}^{2} w_{0}(z)+H(x, y)+\mathrm{i} h_{1}(x, y)+\mathrm{i} C_{0}\left((z \bar{z})^{2}-h_{0}\right),
$$

So, during above argument we had proved that by using our algorithm; we can find the solution of the problems (1.1) and (1.2) with only one constant $C_{0}$, and linearly independent solution for corresponding homogeneous problem is

$$
u_{0}=\mathrm{i}\left((z \bar{z})^{2}-h_{0}\right),
$$

Here $h_{0}$ is bi-harmonic function and uniquely determined from problem (2.12).

\section{Numerical Solution}

Let us divide the rectangle $D$ by $N-1$ straight lines, parallel to coordinate axes and for simplicity the nodes are equidistant which denoted by:

$$
\begin{aligned}
& x_{k}=a+k h, h=\frac{b-a}{N}, k=0, \cdots, N . \\
& y_{j}=c+j h, h=\frac{d-c}{N}, j=0, \cdots, N .
\end{aligned}
$$

Let's consider Dirichlet problem for tri-harmonic Equation (2.2). The tri-harmonic equation is a sixth order elliptic partial differential equation:

$$
\Delta^{3} U=\frac{\partial^{6} U}{\partial x^{6}}+3\left(\frac{\partial^{6} U}{\partial x^{4} \partial y^{2}}+\frac{\partial^{6} U}{\partial x^{2} \partial y^{4}}\right)+\frac{\partial^{6} U}{\partial y^{6}},
$$

which is encountered in viscous flow problems.

In the general case, poly-harmonic equation $\Delta^{k} U=f$, in the discrete setting, are usually solved by applying a Laplacian discretization repeatedly on a mesh, with the cotangent formula being the most popular and the one used in [7]. Although this formulation is efficient and particularly useful in interactive applications, it supports only one particular way of specifying boundary conditions and the results often have significant mesh dependence. The convergent discretization based on a quadratic fitting scheme could be found in [8]. Anyway, Dang Q. A. [9] studied an iterative method for solving a BVP for a tri-harmonic type equation based on using boundary domain operator defined on pairs of boundary and domain functions in combination with parametric extrapolation technique and reduces the BVP for sixth order equation to a sequence of BVPs for Poisson equation.

Next, For finding numerical solution of Poincare problems (2.5) and (2.6); we solve it in domain with the boundary $\Gamma^{\prime}$, whereas

$$
\begin{aligned}
\Gamma^{\prime}=\left\{\left(x_{i}, y_{j}\right): x_{i}=a+h, b-h,\right. \\
\left.y_{j}=c+h, d-h \quad 1 \leq i, j \leq N-1,\right\}
\end{aligned}
$$

where the values of $\Delta_{h}^{2} U_{h}$ are known on the boundary 
points $\Gamma^{\prime}$. Because of simplicity and getting rid of complexity of the system, we may assume two points in (2.5) on the top left and down right corner of the boundary $\Gamma^{\prime}$ are fixed, i.e.: $\left(x_{0}, y_{0}\right)=(a+h, d-h)$ and $\left(x_{1}, y_{1}\right)=(b-h, c-h)$,

so:

$$
u_{1}\left(x_{0}, y_{0}\right)=u_{10}=0, u_{1}\left(x_{1}, y_{1}\right)=u_{11}=0,
$$

By denote the values of function $u_{1}$ in the mesh points approximately:

$$
\left(u_{1}\right)_{k}^{j} \approx u_{1}(k, j), \quad k, j=0, \cdots, N
$$

and from the grid boundary conditions:

$$
\frac{\left(u_{1}\right)_{i+1}^{j}-\left(u_{1}\right)_{i}^{j}}{h}=\frac{1}{4}\left(\Delta_{h}^{2} U_{h}\right)_{i}^{j}, \quad i, j=1, \cdots, N-1
$$

we will find the values in the grid points on the sides of $\Gamma^{\prime}$ parallel to $\mathrm{OX}$ axis.

Finally, the values of $\left(u_{1}\right)_{i}^{j}$ inside $\Gamma^{\prime}$ and on the sides $\Gamma^{\prime}$ parallel to OY axis will be found from the system of linear equation, main matrix of this system may be reduced to the tri-diagonal form :

$$
T=\left[\begin{array}{cccccc}
A & B & 0 & \ldots & \ldots & 0 \\
B & A & B & 0 & \ldots & 0 \\
0 & B & A & B & \ldots & 0 \\
\vdots & \vdots & \vdots & \vdots & \ddots & \vdots \\
\vdots & \vdots & \vdots & \vdots & \ddots & \vdots \\
0 & 0 & 0 & 0 & \ldots & A
\end{array}\right],
$$

where:

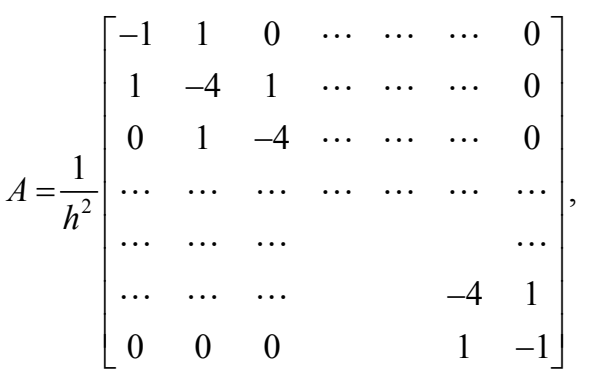

$$
\begin{aligned}
& B=\frac{1}{h^{2}}\left[\begin{array}{cccccc}
0 & 0 & 0 & \cdots & 0 & 0 \\
0 & 1 & 0 & \cdots & 0 & 0 \\
0 & 0 & 1 & \cdots & 0 & 0 \\
\cdots & \cdots & \cdots & \cdots & \cdots & \cdots \\
0 & 0 & 0 & \cdots & 1 & 0 \\
0 & 0 & 0 & \cdots & 0 & 0
\end{array}\right],
\end{aligned}
$$

the matrix $T$ is diagonally dominant, therefore this linear system is uniquely solvable; so considered modified Poincare problem is uniquely solvable too and then from maximum principle [10], we have unique solvability of this system.

Next, we consider the problem (2.10). A fast solver for compact discretizations of the bi-harmonic problem was presented in [11-13], also Dang Q. A. in [14,15] with the help of boundary or mixed boundary-domain operators introduced appropriately, constructed iterative methods for bi-harmonic and bi-harmonic type equations associated with the Dirichlet, Neumann or simple type of mixed boundary conditions.

By finite difference method, we find the values of the function $H$ in the mesh points approximately:

$$
H_{i}^{j} \approx H(i, j), \quad i=1, \cdots, m, \quad j=1, \cdots, n .
$$

we use the discrete analogue of the Laplace operator:

$$
\Delta_{h}^{2} H_{h}=\frac{1}{h^{2}}\left(H_{i+1}^{j}-4 H_{i}^{j}+H_{i-1}^{j}+H_{i}^{j+1}+H_{i}^{j-1}\right),
$$

and may discretisize biharmonic equation in (2.10) as

$$
\begin{aligned}
\Delta_{h} \Delta_{h} H_{h}= & \frac{20}{h^{4}} H_{i}^{j}-\frac{8}{h^{4}}\left(H_{i-1}^{j}+H_{i+1}^{j}\right) \\
& -\frac{8}{h^{4}}\left(H_{i}^{j-1}+H_{i}^{j+1}\right) \\
& +\frac{2}{h^{4}}\left(H_{i+1}^{j+1}+H_{i-1}^{j+1}+H_{i+1}^{j-1}+H_{i-1}^{j-1}\right) \\
& +\frac{1}{h^{4}}\left(H_{i+2}^{j}+H_{i-2}^{j}+H_{i}^{j+2}+H_{i}^{j-2}\right), \\
\left.H\right|_{\Gamma}= & \left(\Re\left(f_{0}\right)\right)_{h},\left.\quad\left(\delta_{h} H_{h}\right)\right|_{\Gamma}=\left(f_{1}\right)_{h} .
\end{aligned}
$$

Here forward divided differences for the operator $\frac{\partial}{\partial N}$ in (2.10) noted by $\delta_{h}$, and $\left(\mathfrak{R} f_{0}\right)_{h},\left(f_{1}\right)_{h}$ are values of functions $\left(\mathfrak{R} f_{0}\right), f_{1}$ in the boundary points of mesh.

The discretization obtained here and also in [16], tends to symmetric matrices so the convergence analysis is simpler, but there now exist standard fast numerical algorithms for this kind of boundary value problems too. In [17-19] for the two dimensional case, problem (2.10) has been reduced to second kind integral equations and a fast numerical algorithm is developed based on the constructed SKIE in [20].

The discrete problem (3.1) approximates the problem (2.10) (see [21]), therefore, from the stability of the problem (3.1), we have the convergence of the grid function to $\left\{H\left(x_{i}, y_{j}\right)\right\} \quad[3$, p. 30, Theorem 2.5]. From the last two equation of the (3.1); we find the values of the function $H_{h}$ in the points $\left(x_{i}, y_{j}\right) \quad(i, j=0,1, \cdots, N)$ and if the values $H_{i}^{j}$ are on interior nodes, we find them from the linear system with symmetric pentadiagonal matrix, so from positive definiteness of this matrix, we may prove the stability of the (3.1) and an algorithm for the solution of the system can be found in [22]. 
Therefore the problems (1.1) and (1.2) reduced to six uniquely solvable problems for properly elliptic equations with real coefficients, which were solved by grid methods.

\section{REFERENCES}

[1] N. E. Tovmasyan, "Non-Regular Differential Equations and Calculations of Electromgnetic Fields," World Scientific Publishing Co., Singapore City, 1998.

[2] G. C. Wen, "Approximate Methods and Numerical Analysis for Elliptic Complex Equations," CRC Press Inc., Amsterdam, 1999.

[3] J. W. Thomas, "Numerical Partial Differential Equations Conservation Laws and Elliptic Equations," Springer, New York, 1991.

[4] C. Grossmann, H.-G. Roos and M. Stynes, "Numerical Treatment of Partial Differential Equations," Springer, Berlin, 2007. doi:10.1007/978-3-540-71584-9

[5] J. L. Lions and E. Magenes, "Problemes Aux Limites Non-Homogenes et Applications Vol. 1," Dunod, Paris, 1968.

[6] H. Begehr and T. Vaitekhovich, "Iterative Dirichlet Problem for the Higher Order Poisson Equation," Le Matematiche, Vol. 63, No. 1, 2008, pp.139-154.

[7] M. Botsch and L. Kobbelt, "An Intuitive Framework for Real-Time Freeform Modeling," Siggraph'04: ACM Siggraph 2004 Papers, New York, 2004, pp. 630-634,

[8] G. Xu, "Consistent Approximations of Some Geometric Differential Operators," Technical Report ICM-06-001, Institute of Computational Mathematics and Scientific \& Engineering Computing, Chinese Academy of Sciences, Beijing, 2000.

[9] Q. A. Dang, "Using Boundary-Operator Method for Approximate Solution of a Boundary Value Problem (BVP) for Triharmonic Equation," Vietnam Journal of Mathematics, Vol. 33, No. 1, 2005, pp. 9-17.

[10] L. Collatz, "The Numerical Treatment of Differential Equations," Springer, New York, 1966.

[11] H. Begehr, "Boundary Value Problems in Complex Analysis I," Boletin de la Asociación Matemática Vene- zolana, Vol. 12, No. 1, 2005, pp. 65-85.

[12] H. Begehr, "Boundary Value Problems in Complex Analysis II," Boletin de la Asociación Matemática Venezolana, Vol. 12, No. 2, 2005, pp. 217-250.

[13] P. Peisker, "On the Numerical Solution of the First Biharmonic Equation," Mathematical Modelling and $\mathrm{Nu}$ merical Analysis, Vol. 22, No. 4, 1988, pp. 655-676.

[14] Q. A. Dang, "Boundary Operator Method for Approximate Solution of Biharmonic Type Equation," Vietnam Journal of Mathematics, Vol. 22, No. 1-2, 1994, pp. 114120.

[15] Q. A. Dang, "Mixed Boundary-Domain Operator in Approximate Solution of Biharmonic Type Equation," Vietnam Journal of Mathematics, Vol. 26, No. 3, 1998, pp. 243-252.

[16] Q. A. Dang, "Iterative Method for Solving the Neumann Boundary Value Problem for Biharmonic Type Equation," Journal of Computational and Applied Mathematics, Vol. 196, No. 2, 2006, pp. 634-643. doi:10.1016/j.cam.2005.10.016

[17] S. D. Jiang, B. Ren, P. Tsuji and L. X. Ying, "Second Kind Integral Equations for the First Kind Dirichlet Problem of the Biharmonic Equation in Three Dimensions," Journal of Computational Physics, Vol. 230, No. 19, 2011, pp. 7488-7501. doi:10.1016/j.jcp.2011.06.015

[18] I. N. Vekua, "New Methods for Solving Elliptic Equations," John Wiley, New York, 1967.

[19] J. Cohen and J. Gosselin, "The Dirichlet Problem for the Biharmonic Equation in a C1 Domain in the Plane," Indiana University Mathematics Journal, Vol. 32, No. 5, 1983, pp. 635-685. doi:10.1512/iumj.1983.32.32044

[20] P. Bjorstad, "Fast Numerical Solution of Thew Biharmonic Dirichlet Problem on Rectangles," SIAM Journal on Numerical Analysis, Vol. 20, No. 1, 1983, pp. 59-71. doi:10.1137/0720004

[21] S. G. Mikhlin, "Integral Equations," Pergamon Press, New York, 1957.

[22] M. H. Koulaei and F. Toutounian, "Factored Sparse Approximate Inverse of Block Tridiagonal and Block Pentadiagonal Matrices," Applied Mathematics and Computation, Vol. 184, No. 2, 2007, pp. 223-234. doi:10.1016/j.amc.2006.05.204 